\title{
Segmentation of areas affected by Keratosis Epidermoid Carcinoma in dermatoscopic images
}

\author{
J. C. Martínez-Perales ${ }^{\# 1}$, R. Flores-Carapia ${ }^{* 2}$, B. Luna-Benoso ${ }^{\# 3}$

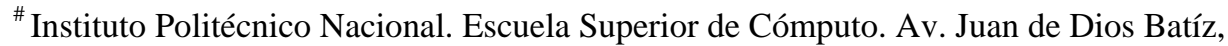 \\ esq. Miguel Othón de Mendizábal, México City 07738, México. \\ 1 pepe_cruzm@hotmail.com
${ }^{3}$ blunabenoso@yahoo.com \\ * Instituto Politécnico Nacional. Centro de Innovación y Desarrollo Tecnológico en Cómputo. Av. Juan de Dios \\ Batíz, esq. Miguel Othón de Mendizábal, México City 07738, México. \\ 2rfcarapia@yahoo.com
}

Abstract-Skin cancer is the most frequent in humans, this is divided into two ample groups: Nonmelanoma skin cancer and melanoma. Epidermoid carcinoma is a type of non-melanoma cancer and is the second most frequently after basal cell carcinoma. Epidermoid carcinoma is a malignant neoplasm derived from the cells of the epidermis or its annexes, with the capacity to produce metastasis. This work presents a methodology to obtain the segmentation of the area affected by epidermoid carcinoma in dermatoscopic images. The methodology is based on methods in the spatial domain.

Keyword - Image analysis, image segmentation, keratosis epidermoid carcinoma, dermatoscope.

\section{INTRODUCTION}

Skin cancer includes the type of melanoma cancer and the type of non-melanoma cancer, the type of cancer being the most common non-melanoma skin cancer in the world with approximately $40 \%$ of all types of cancer [4]. In the United States, approximately 3.5 million cases have been reported in 2006 [2], and more than one million new cases and 1000 deaths in 2009 [3]. It is estimated that each year between 2 and 3 million of new cases of non-melanoma type skin cancer arise, where women younger than 45 years are the prone [1]. Exposing to UV radiation is considered the main risk factor for the appearance and development of non-melanoma skin cancer [5]. The most common types of non-melanoma skin cancer presented in people are basal cell carcinoma and squamous cell carcinoma or carcinoma epidermoid or squamous cell $[6,7,8]$. The behavior of epidermoid carcinoma is more aggressive than that of basal cell carcinoma, appearing in its early phases in skin, later in lymph nodes, and in rare cases with metastasis to other organs [8]. Epidermoid carcinoma is a malignant tumor whose origin is from epidermal keratinocytes and other epithelia. Generally the size of the tumor (greater horizontal diameter) is directly related to a higher recurrence rate of the neoplasia, of regional metastasis and with lower survival [9]. In medical terms of Mexican dermatology, the epidermoid carcinoma is divided into 5 types: 1) ulcerous, 2) nodular, 3) nodular keratosis, 4) vegetative and 5) superficial [10]. The most frequent is the ulcerous, next is the nodular type that is characterized by an exophytic lesion of super smooth muscle. The keratosis nodular variant presents as a nodular neoplasm type or plaque presenting in the form of a cutaneous horn, the base of this lesion is presented a raised erythematous border of firm consistency. The vegetation type generally starts as a nodular lesion at the center of which the lesion develops, this type is usually larger. On the other hand, the super-type develops as an erythematous plaque, keratosic or verrucous. Studies carried out in Mexico show that this type of neoplasias come about more frequently in women, with an average age of 71 years and predominantly on the face, these lesions are present on average within a radius of $3 \mathrm{~cm}$ and most present ulcerations [8]. The epidermoid carcinoma of the keratosis type (which is the one discussed in this paper) is fast growing, mostly asymptomatic, destructive and infiltrating, characterized by presenting a scaly surface of yellowish white, blood stains and whitish areas with no structure, usually present on the hands, legs and face (figure 1).

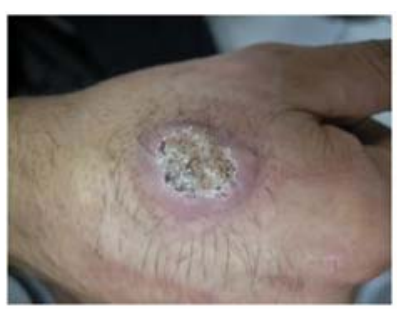

a)

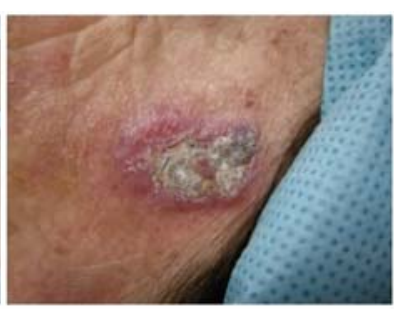

b)

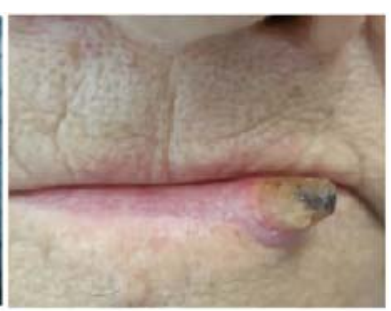

c)

Fig. 1. Keratosis epidermoid carcinoma presented in a) hand, b) face and c) lip. 
Currently, systems have been developed as CAD (computer-aided design) in different areas of medicine with the aim of supporting the pre-diagnosis of the type of disease in question. Regarding the type of epidermoid carcinoma, CAD systems have been developed that allow the doctors to assist in decision making, or in detecting the type of neoplasia, or in detecting tumor recurrence $[11,12,13]$. This work shows a methodology that allows to obtain the segmentation of the zone affected by epidermoid carcinoma of the nodular keratosis type, for this purpose, dermatoscopic images are used which are applied methods in the spatial domain.

\section{Methodology}

\section{A. Obtaining the image}

One commonly used technique for the clinical diagnosis of epidermoid carcinoma is the dermatoscopy, which consists in making use of an optic instrument called dermatoscope, which enlarges the lesion image to better examine the affected area below the cutaneous surface. Figure 2 shows the image of the area affected by cell carcinoma and the right side shows the image of the same affected area seen through a dermatoscope.
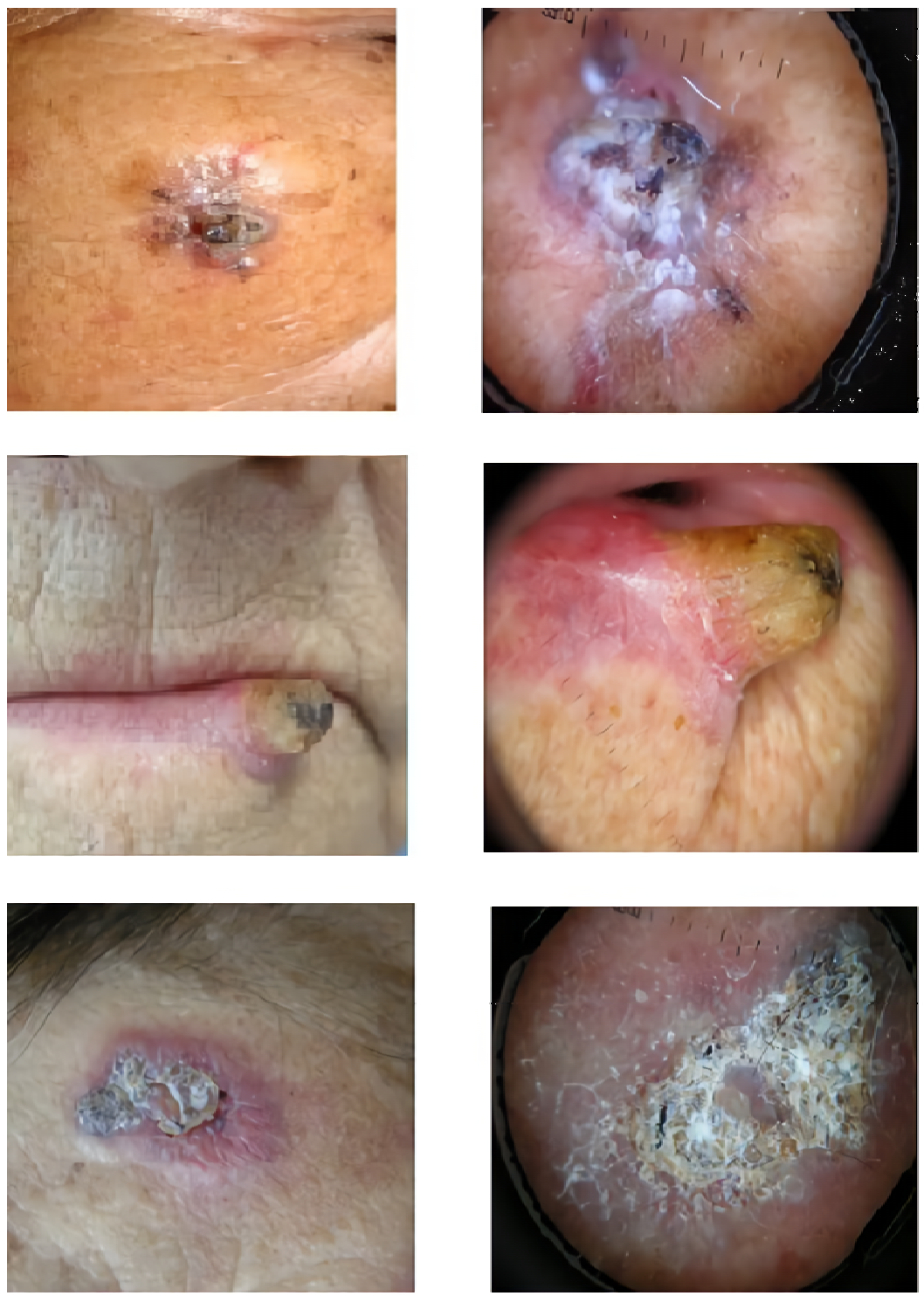

Fig. 2. Keratosis epidermoid carcinoma, in the left column shows the affected area and in the right column view with a dermatoscope.

\section{B. Digital image}

It is necessary to mention the definition of what is a digital image. A digital image is a two-dimensional function $f(x ; y)$ of the light intensity (brightness) at a point in space, where $(x ; y)$ is the coordinates of that point considering the origin in the upper left part of the image [14]. A digital image is a function $f(x ; y)$ discretized in both spatial coordinates and brightness, it is often often represented as a two-dimensional matrix $F_{i j}=$ $\left(f_{i j}\right)_{H \times W}$, where $H$ and $W$ represent the size of the image, (referring $H$ and $W$ to high and width of the image respectively) with $f_{i j}=f\left(x_{i}, x_{j}\right)$ (figure 3 ). 


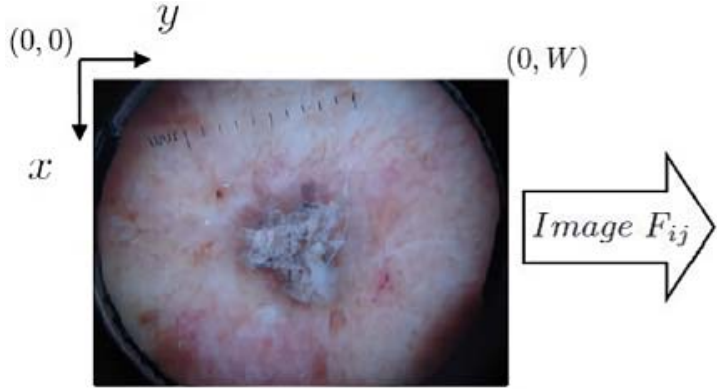

$(H, 0)$

$(H, W)$

\begin{tabular}{|c|c|c|c|c|c|}
\hline$f(0,0)$ & $f(0,1)$ & $f(0,2)$ & $f(0,3)$ & $\ldots$ & $f(0, W)$ \\
\hline$f(1,0)$ & $f(1,1)$ & $f(1,2)$ & $f(1,3)$ & $\ldots$ & $f(1, W)$ \\
\hline$f(2,0)$ & $f(2,1)$ & $f(2,2)$ & $f(2,3)$ & $\ldots$ & $f(2, W)$ \\
\hline$f(3,0)$ & $f(3,1)$ & $f(3,2)$ & $f(3,3)$ & $\ldots$ & $f(3, W)$ \\
\hline$f(4,0)$ & $f(4,1)$ & $f(4,2)$ & $f(4,3)$ & $\ldots$ & $f(4, W)$ \\
\hline$f(5,0)$ & $f(5,1)$ & $f(5,2)$ & $f(4,3)$ & $\ldots$ & $f(5, W)$ \\
\hline$\vdots$ & $\vdots$ & $\vdots$ & $\vdots$ & $\ldots$ & $\vdots$ \\
\hline$f(H, 0)$ & $f(H, 1)$ & $f(H, 2)$ & $f(H, 3)$ & $\ldots$ & $f(H, W)$ \\
\hline
\end{tabular}

Fig. 3. Digital image.

The proposed methodology was divided into eight modules. Each module makes use of methods in the domain space. The resulting image in each of the modules is used later in some other module, so it was decided to carry out an enumeration that would be as clear as possible. Next is presented each of the modules that make up the methodology.

Module 1: Figure 4 shows the first processing performed on the set of images dermatoscopies. In order as are showed the arrows in the image: 4-a) the image obtained by of a dermatoscopy of the affected area. In 4-b) is showed the image in grayscale generated by the formula: $(R+G+B) * \frac{255}{\max _{i \in I}\left(R_{i}+G_{i}+B_{i}\right)}$, where $\mathrm{R}, \mathrm{G}$ and $\mathrm{B}$ represents the decomposition of the three planes of the image. In 4-c) we apply a statistical filter of minimum order, these are non-linear spatial filters based on an ordering of pixels in a neighborhood, in this case selects the smallest value of an ordered window of gray level values. In 4-d) the image with a threshold of 64 is binarized, thus obtaining in the black area the background of the dermoscopic image. 4-e) shows the image by placing a frame with a thickness of 30 pixels. In 4-f) noise shown in the blank area is eliminated, this took I just pocketing the connected component of greater area. Subsequently, the area with the largest area was selected, and the area was placed in white and the rest in black to finally obtain the image In 4-g) which we will refer to later as $m 0$.

a)

b)

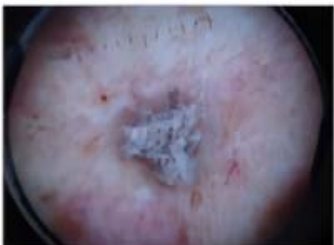

c)

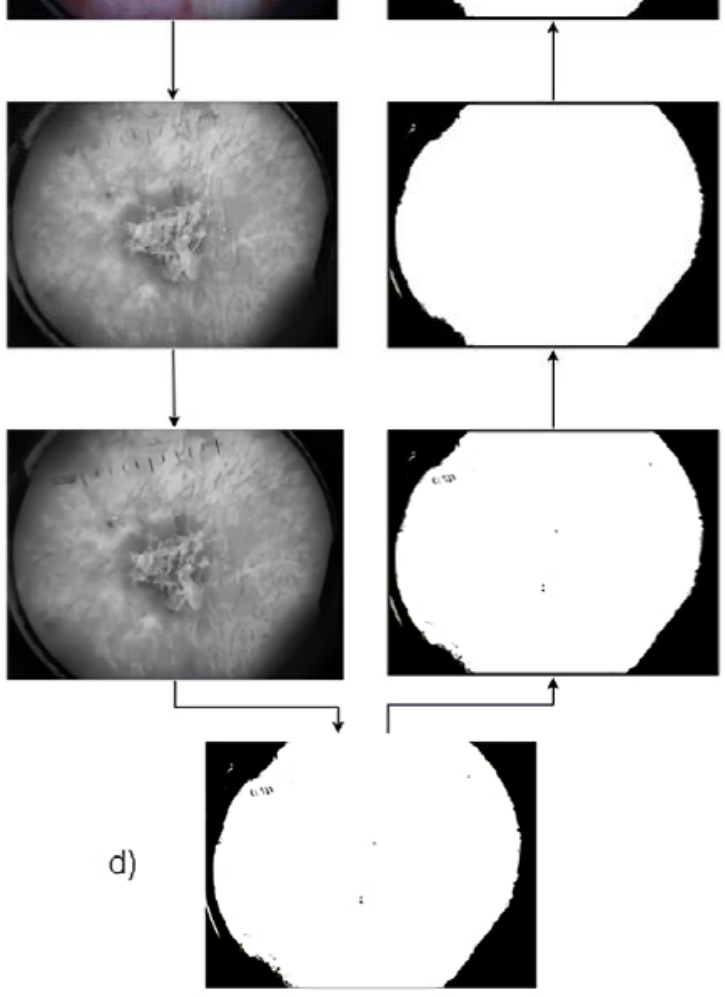

Fig. 4. Module 1 of the digital image processing. g) $\mathrm{mo}$

f)

e)
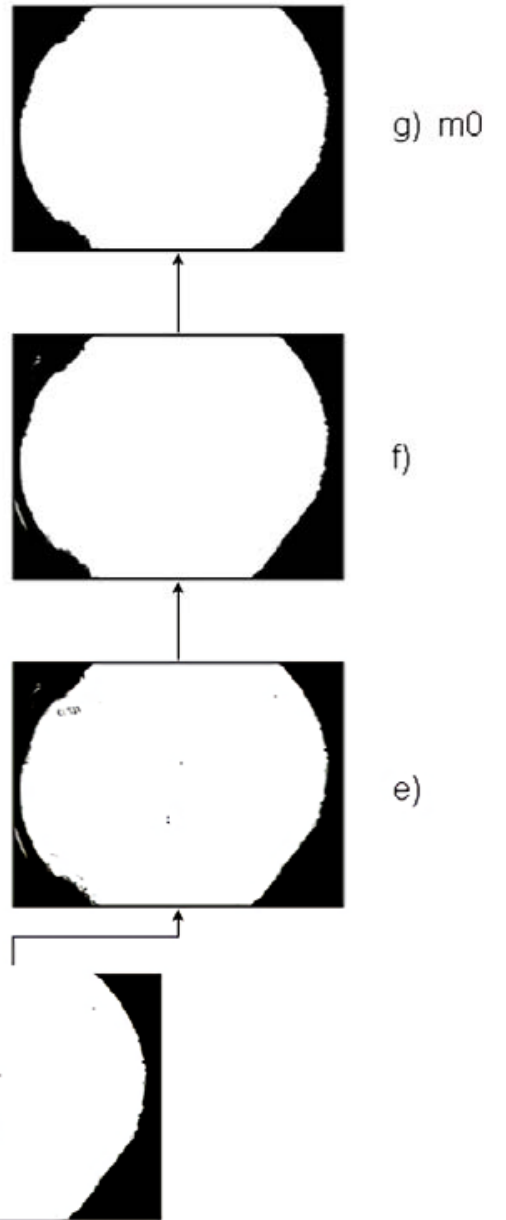
Module 2: The following module of the image analysis is shown in figure 5. Starting from the dermatoscopic image, 5-D1: shows the image in grayscale by applying the formula with 4-b). 5-D2: 4 was subsequently applied to a Gaussian filter. 5-D3: we immediately applied Sobel operator using a mask of $5 \times 5$. Moreover, starting from the dermoscopic image, 5-R1: shows the image in grayscale considering only the red channel. 5-R2: a Gaussian filter was subsequently applied. 5-R3: and again the operator Sobel with mask of $5 \times 5$.

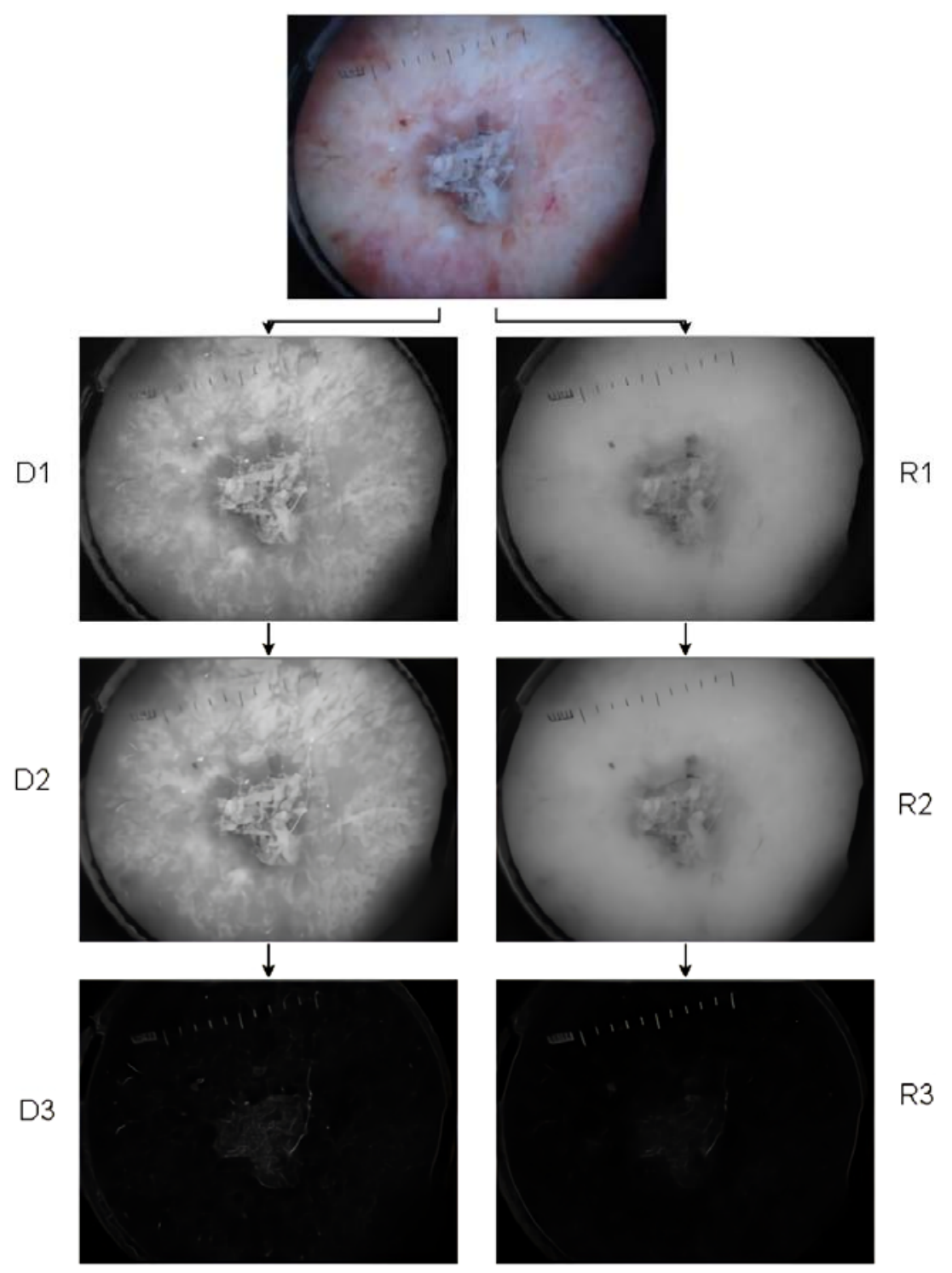

Fig. 5. Digital image processing module 2.

Module 3: The following module of the digital image processing is shown in figure 6 as an example. In order by arrows. 6-R2: Result of applying Gaussian filter to R1. 6-M1: detection of edges by means of mathematical morphology, to say, if $I$ is the original image and $G$ the resulting image, then $G=(I \oplus b)-(I \ominus b)$ where the operators $\bigoplus$ and $\ominus$ represent the dilation and erosion in images grayscale and $b$ is the structural element. 6-M2: shows the result of applying the logical operation M1 AND m0. 6-M3: Result of applying the morphological lock operation to 6-M2. 6-M4: Expanding the 6-M3 histogram. 


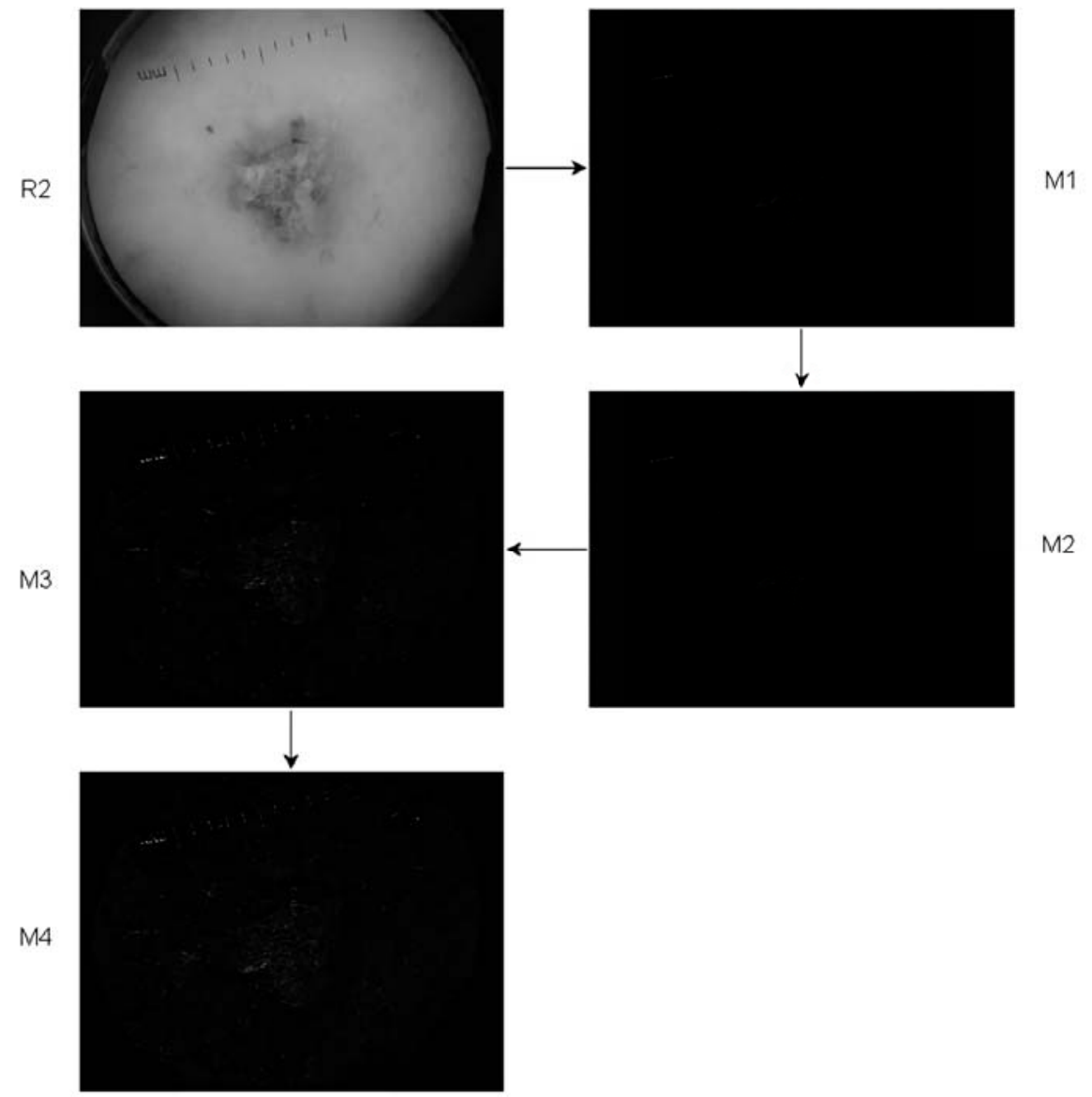

Fig. 6. Module 3 of the digital image processing.

Module 4: Figure 7 shows the next step of image processing. In order to as appear according to the arrows. 7D3: the operator Sobel was applied to D2 with mask extension of $5 \times 5$. 7-R3: the Sobel operator was applied to R2 with mask extension of $5 \times 5$. 7-V1: result of adding the images D3 and R3. 7-M4: expansion of the histogram of M3. 7-V2: result of adding the images V1 and M4. 


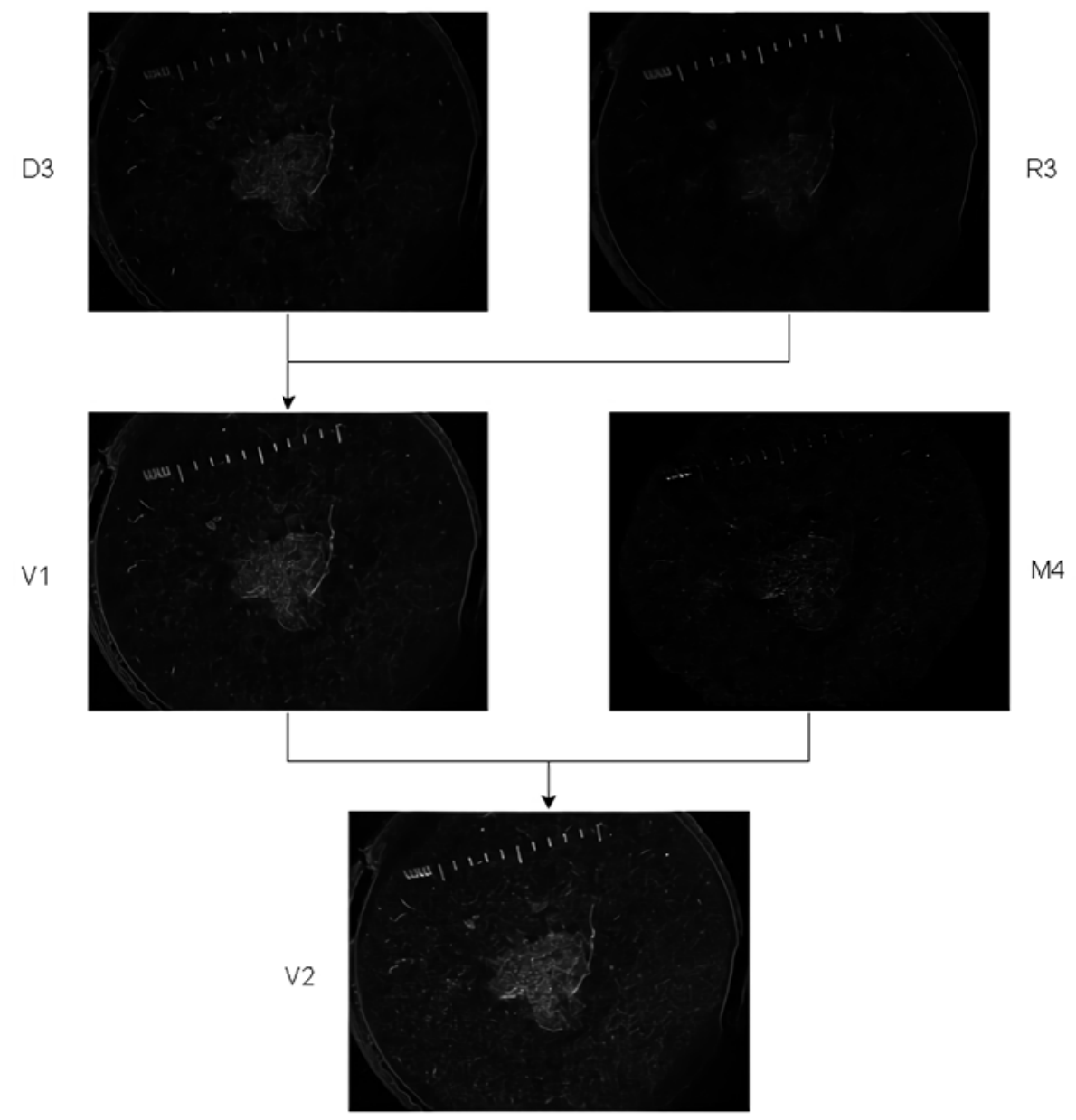

Fig. 7. Module 4 of the digital image processing.

Module 5: Figure 8 shows the next step of the digital processing of the image. In order to as appear the following arrows: 8-V2: Result of adding the images V1 and M4. 8-V3: Logical operation V2 AND m0. 8-V4: thresholding of V3 with threshold 64. 8-V5: morphological operation lock from 11 x 11 to V4. 8-V6: morphological operation lock from 9 x 9 to V5. 8-A1: Logical operation NOT of V6. 


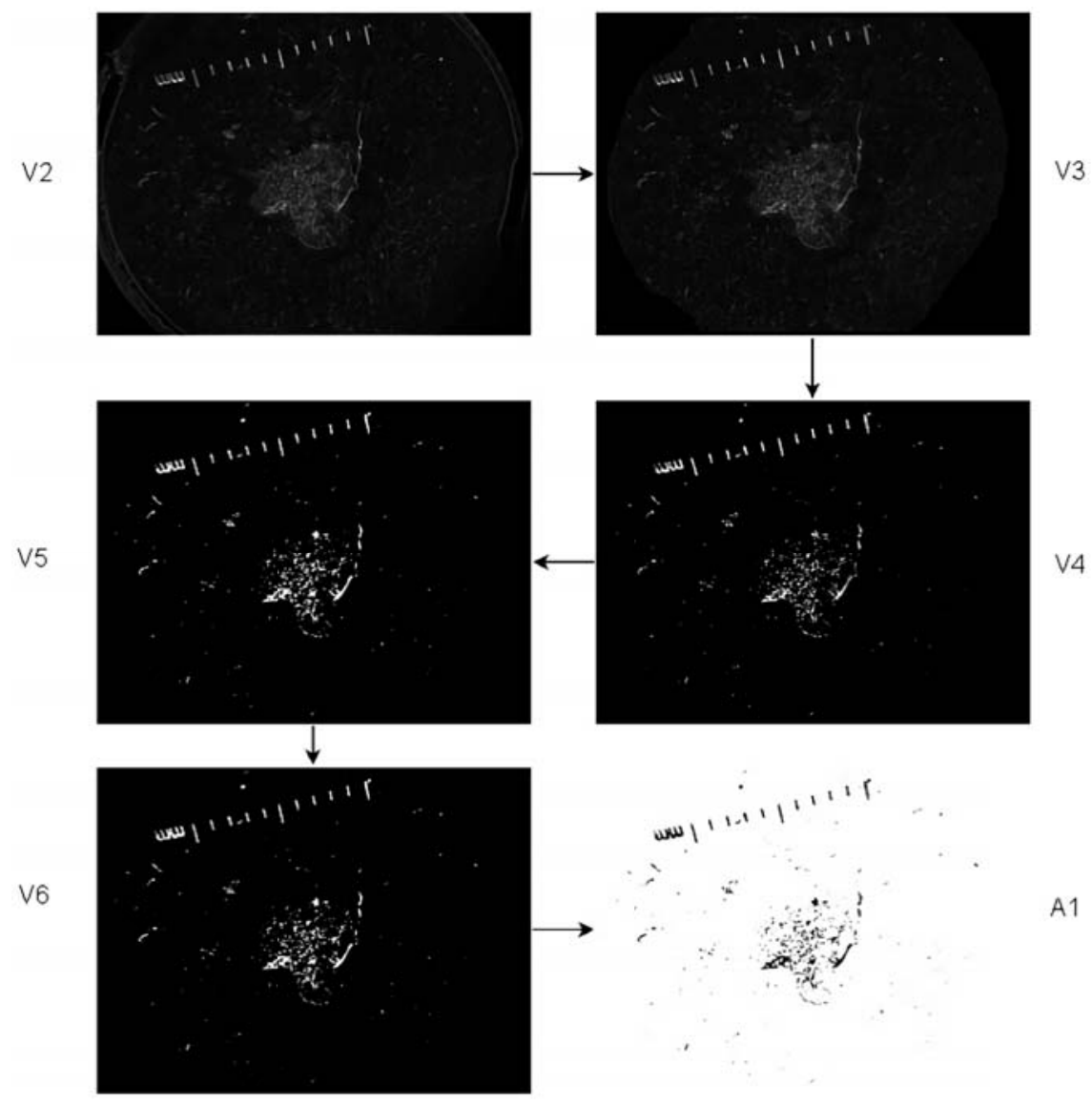

Fig. 8. Module 5 of the digital image processing.

Module 6: Figure 9 shows the sixth module of the digital processing. Following the order of the arrows: 9-A1: shows the logical operation NOT of V6. 9-A2: logical AND operation of A1 with the image original. 9-A3: supercritical approximation with A2. 9-A4: Statistical filter from medium to A3.

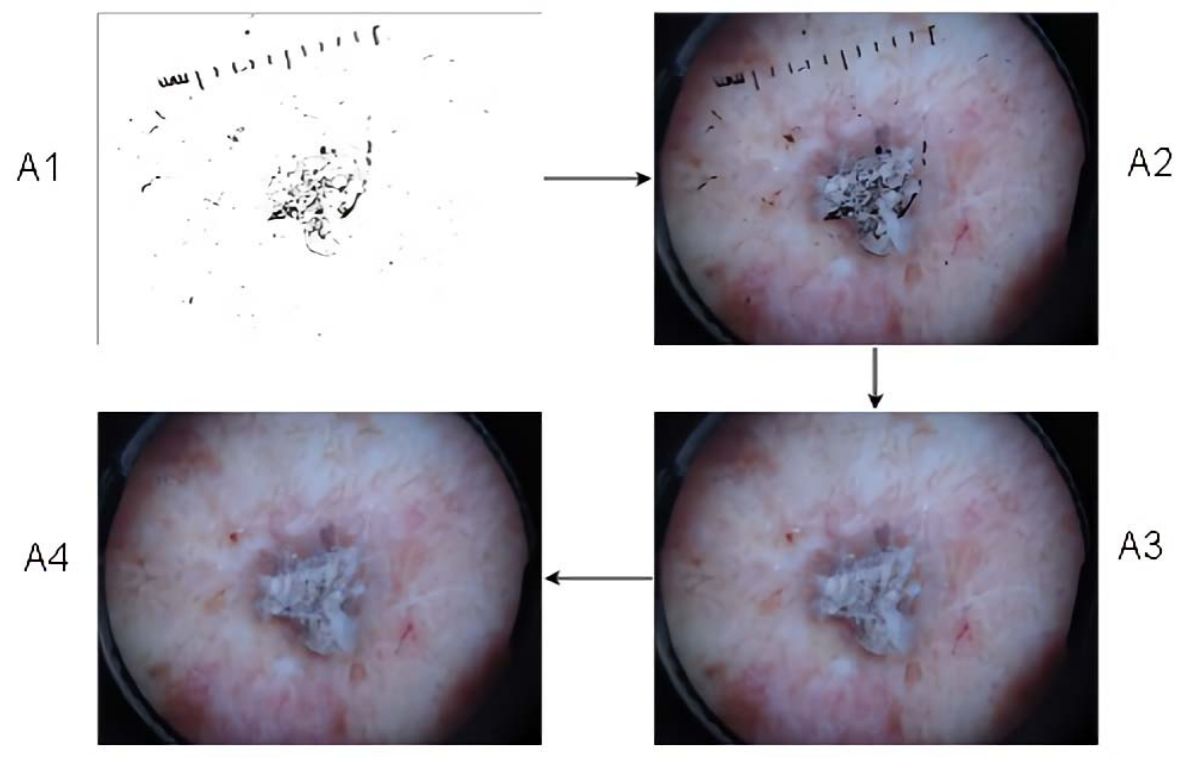

Fig. 9. Module 6 digital processing of the image.

Module 7: Figure 10 shows the seventh module of the image analysis. In order to show the images in the arrows. 10-A4: Statistical filter of median of A3. 10-SG1: gray scale of A4. 10-SG2: Gaussian filtering of SG1. 10-SG3: Sobel operator of D2 with 3 × 3 mask. 10-SG4: logical operation SG3 AND m0. 10-SG5: expansion of SG4 histogram. 


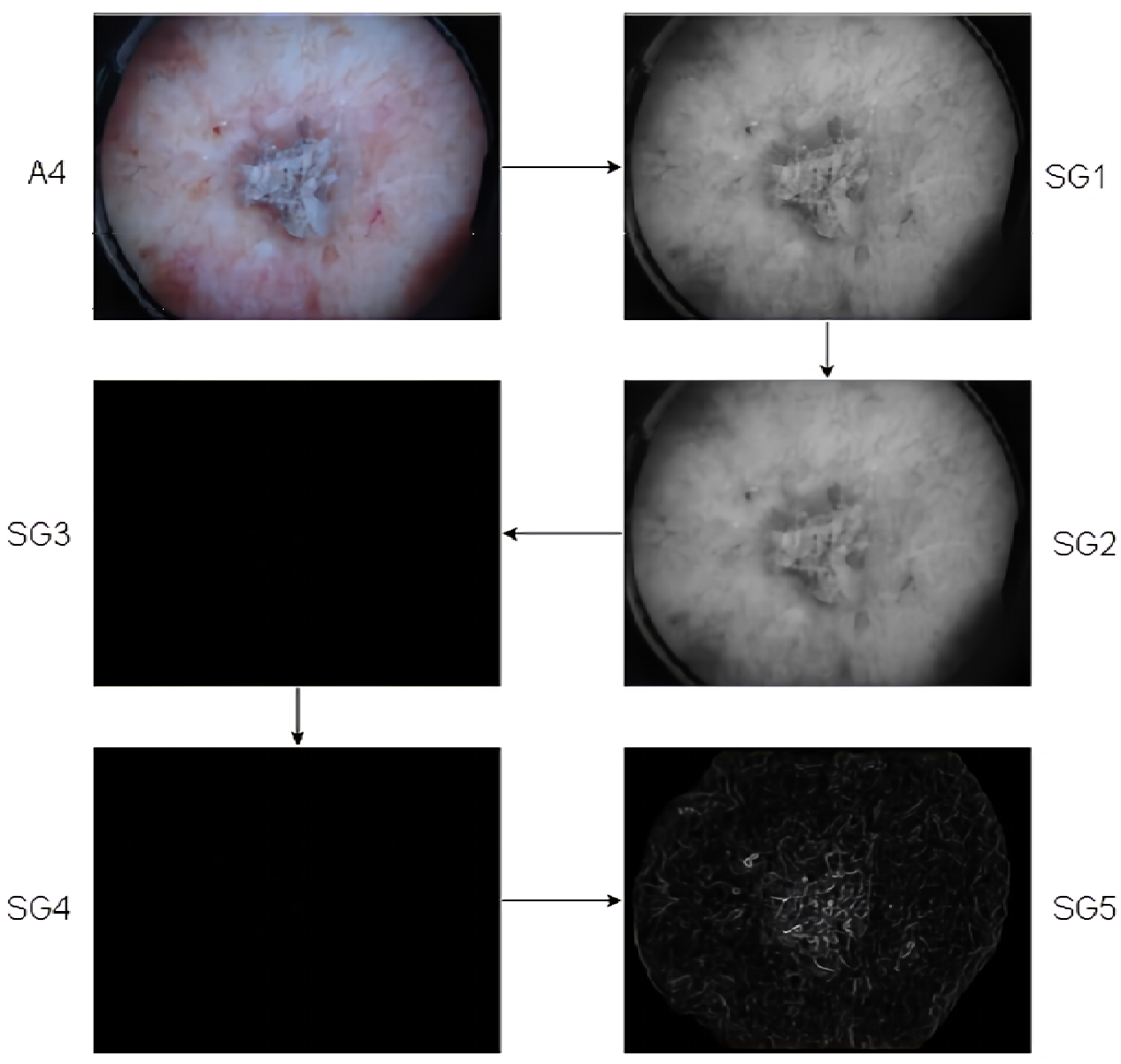

Fig. 10. Image processing digital module 7.

Module 8: Finally figure 11 shows the last module of the digital image processing for to obtain the segmented area affected by the lesion. In order to how the images appear in the arrows. 11-SG5: Expansion of SG4 histogram. 11-SG6: Operation morphological erosion of $3 \times 3$ of SG5. 11-SG7: Ubralization of SG6. 11-SG8: morphological operation dilatation of $3 \times 3$ SG7. 11-SG9: morphological operation erosion with mask of $21 x$ 21 of SG8. 11-SG10: Selection of area with larger size. 


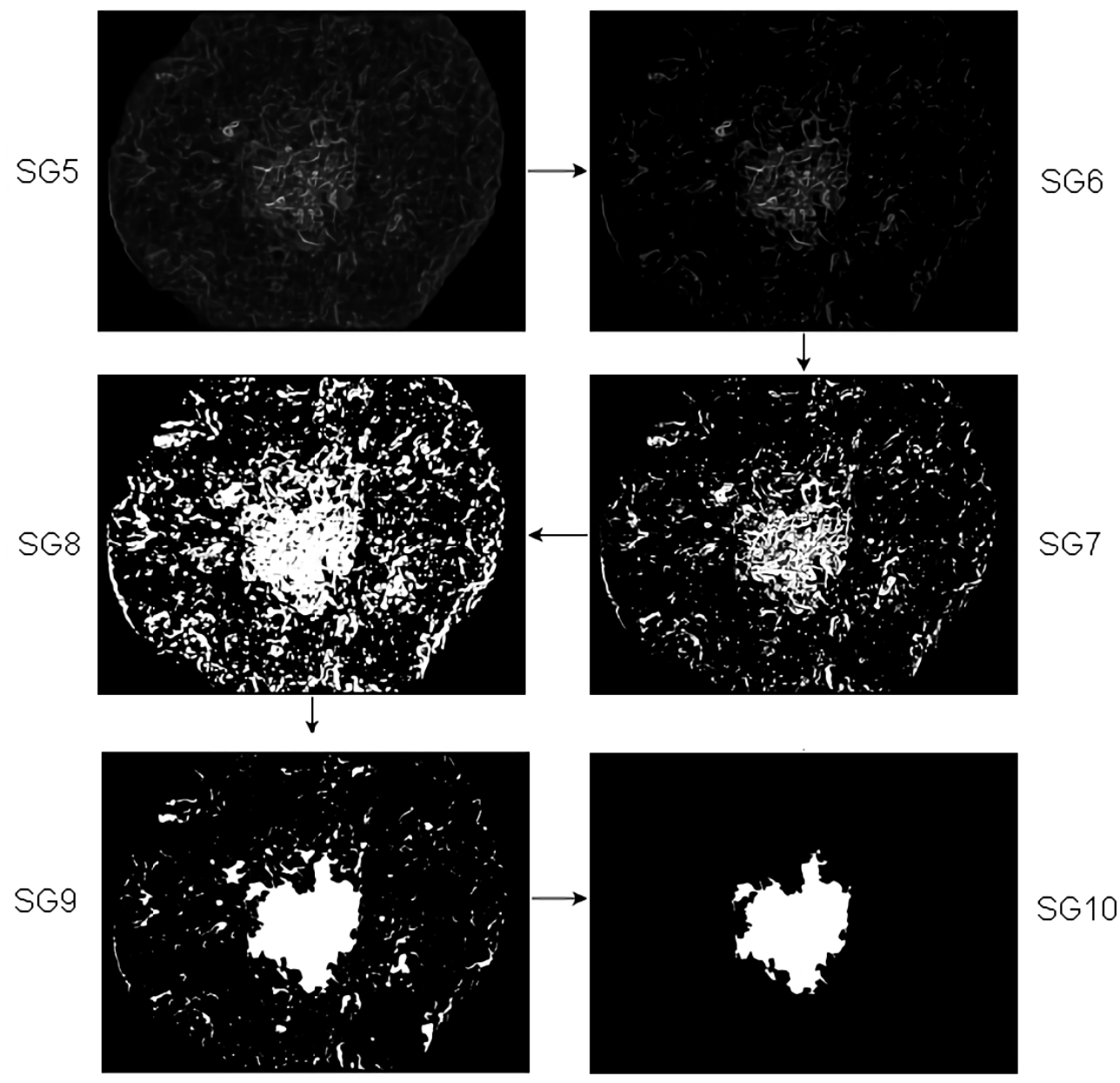

Fig. 11. Module 8 of the digital image processing.

Figures 12 and 13 show two dermoscopic images to which the proposed methodology was applied. In each of the images are observed blocks corresponding to each of the modules that compose the methodology.

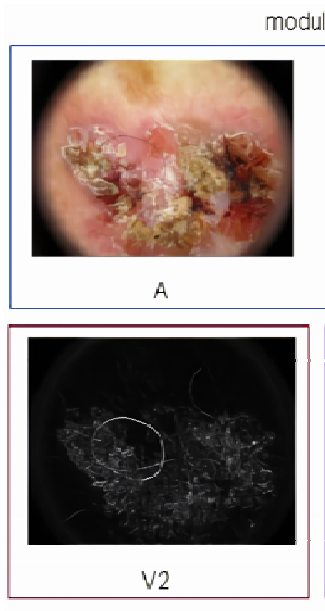

module 4

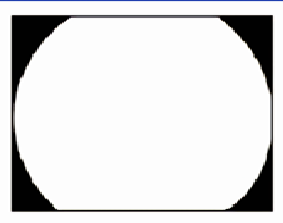

mo

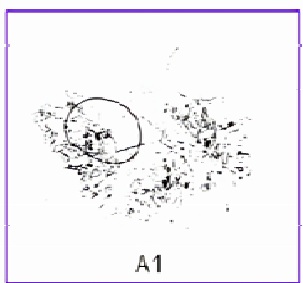

module 5 module 2

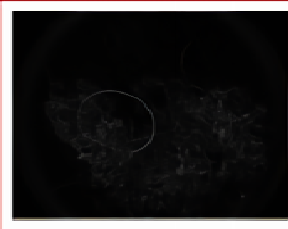

D3

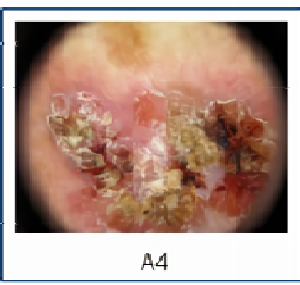

module 6

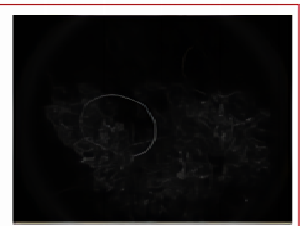

R3

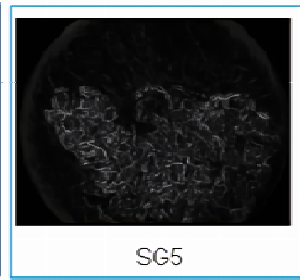

module 7 module 3
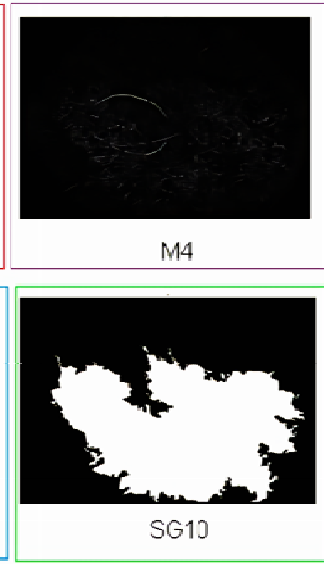

module 8

Fig. 12. Example of the proposed methodology. 

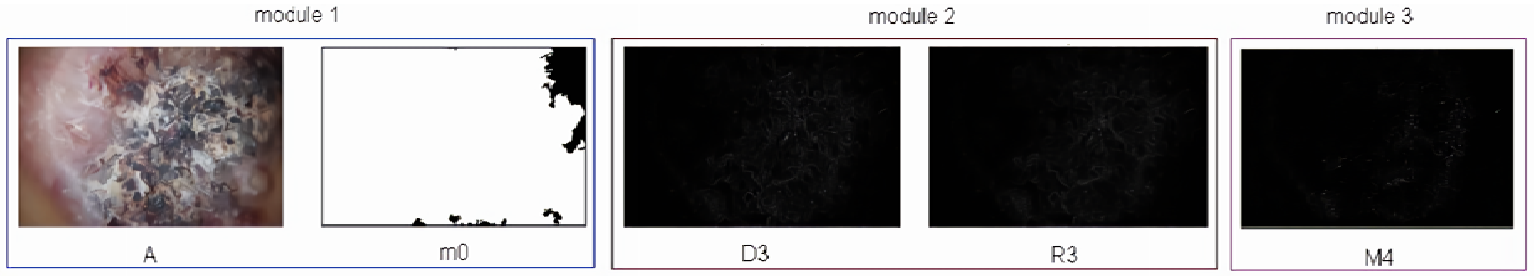

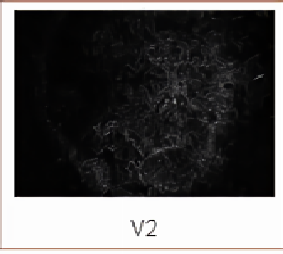

module 4

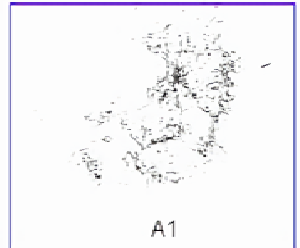

module 5

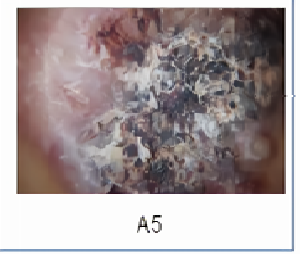

module 6

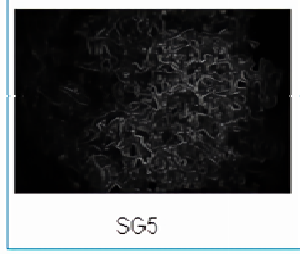

module 7

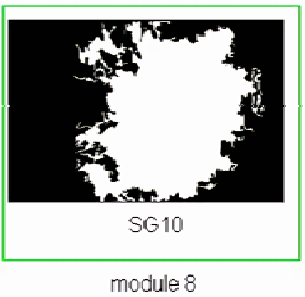

Fig. 13. Example of the proposed methodology.

\section{III.CONCLUSION}

A methodology was proposed to segment images of areas affected by epidermoid carcinoma. A methodology was proposed based on methods in the spatial domain. The images considered were captured by means of a dermatoscope where the affected area is shown. The methodology presented was divided into eight modules. In the first module a binarized image of the circular area corresponding to the skin is obtained, thus eliminating the dark background of the image. In the fifth module the binarized image of the affected area is presented but with noise. Module eight presents the segmented image corresponding to the affected area by epidermoid carcinoma. A total of 30 images were used to test the proposed methodology.

\section{ACKNOWLEDGMENT}

The authors would like to thank the Instituto Politécnico Nacional (Secretaría Académica, EDD, COFAA, SIP, ESCOM), for their economical support to develop this work.on.

\section{REFERENCES}

[1] Z. Al-Dujaili, M. Henry, A.S. Dorizas, N.S. Sadick. Skin cancer concerns particular to women. International Journal of Women's Dermatology, Volume 3, Issue 1, Supplement, pp. 49-51, March 2017.

[2] Ethan Walker, Margaret Mann, Kord Honda, Allison Vidimos, James P. Basilion. Rapid visualization of nonmelanoma skin cancer. Journal of the American Academy of Dermatology, Volume 76, Issue 2, pp. 209-216, Feb. 2017.

[3] Aylin Türel Ermertcan, Peter W. Hellings, Cemal Cingi. Nonmelanoma Skin Cancer of the Head and Neck: Nonsurgical Treatment. Facial Plastic Surgery Clinics of North America, Volume 20, Issue 4, pp. 445-454, Nov. 2012.

[4] Burak Ömür Cakir, Peter Adamson, Cemal Cingi. Epidemiology and Economic Burden of Nonmelanoma Skin Cancer. Facial Plastic Surgery Clinics of North America, Volume 20, Issue 4, pp. 419-422, Nov. 2012.

[5] R Randie H. Kim, April W. Armstrong. Nonmelanoma Skin Cancer. Dermatologic Clinics, Volume 30, Issue 1, pp.125-139, January 2012.

[6] Navid Noroozi, Ali Zakerolhosseini. Computer assisted diagnosis of basal cell carcinoma using Ztransform features. Journal of Visual Communication and Image Representation, Volume 40, Part A, pp.128-148, Oct. 2016.

[7] Martin Ulrich, Iris Zalaudek, J. Welzel. Shining into the White: The Spectrum of Epithelial Tumors from Actinic Keratosis to Squamous Cell Carcinoma. Dermatologic Clinics, Volume 34, Issue 4, pp. 459-467, Oct. 2016.

[8] Judith Domnguez-Cherit, Georgina Rodrguez-Gutirrez, Vernica Narvez Rosales, Sonia Toussaint Caire, Vernica Fonte Avalos. Caractersticas del carcinoma epidermoide cutaneo y riesgo para el desarrollo de recidivas con cirugía convencional y cirugía con transoperatorio tardo. Ciruga y Cirujanos, In press, corrected proof, Available online 10 January 2017DCA12-70 data sheet," Opto Speed SA, Mezzovico, Switzerland.

[9] A. Nuo-Gonzalez, F.J. Vicente-Marín, F. Pinedo-Moraleda, J.L. López-Estebaranz. Carcinoma epidermoide cutáneo de alto riesgo. Actas Dermo-Sifiliográficas, Volume 103, Issue 7, pp. 567-578, Sep. 2012.

[10] Barrn-Tapia, T., Peniche-Rosado, J., Peniche-Castellanos, A., Arellano-Mendoza, I., Len-Dorantes, G. y Mercadillo-Prez, P. Carcinoma epidermoide de piel en la población mexicana, Rev Med Hosp Gen Mex, 67: 71-77, 2004.

[11] Jinglin Zhou, Bin Xu, Jing Huang, Xiangming Jia, Wei Li. 1H NMR-based metabonomic and pattern recognition analysis for detection of oral squamous cell carcinoma. Clinica Chimica Acta, Volume 401, Issues 12, pp. 8-13, March 2009.

[12] J. Levi Chazen, Christine M. Glastonbury Total laryngectomy for squamous cell carcinoma: recognizing disease patterns to aid detection of tumor recurrence. Clinical Imaging, Volume 38, Issue 5, pp. 659-665, Sep. 2014.

[13] Zhi-Hua Zhou, Yuan Jiang, Yu-Bin Yang, Shi-Fu Chen. Lung cancer cell identification based on artificial neural network ensembles.

[14] González R, Woods J. (2008). Digital image processing, 3rd edn. Prentice Hall, New Jersey.

\section{AUTHOR PROFILE}

José Cruz Martínez Perales: Master in innovation and educational technology by the Institute of University Studies, Puebla Mexico, areas of interest: pattern recognition, cellular automata and neuro linguistic programming.

Benjamín Luna Benoso: Doctor in computer science by the Center of Research in computer Science, areas of interest: pattern recognition, cellular automata, quantum programming, etc. 